\title{
Le parasite hongrois sophocléen de Péter
} Bornemisza

The sophoclean Hungarian parasite of Péter Bornemisza

Bornemisza szophoklészi magyar parazitája

\section{András Kányádi}

\section{(2) OpenEdition}

Journals

Édition électronique

URL : https://journals.openedition.org/efo/4226

DOI : $10.4000 /$ efo. 4226

ISSN : 2275-1947

\section{Éditeur}

INALCO

Édition imprimée

Date de publication : 1 janvier 2014

ISBN : 978-2-343-05394-3

ISSN : 0071-2051

\section{Référence électronique}

András Kányádi, « Le parasite hongrois sophocléen de Péter Bornemisza », Études finno-ougriennes [En ligne], 46 | 2014, mis en ligne le 15 octobre 2015, consulté le 08 juillet 2021. URL : http://

journals.openedition.org/efo/4226; DOI : https://doi.org/10.4000/efo.4226

Ce document a été généré automatiquement le 8 juillet 2021.

\section{(c) (7) (8)}

Études finno-ougriennes est mis à disposition selon les termes de la Licence Creative Commons Attribution - Pas d'Utilisation Commerciale 4.0 International. 


\title{
Le parasite hongrois sophocléen de Péter Bornemisza
}

\author{
The sophoclean Hungarian parasite of Péter Bornemisza \\ Bornemisza szophoklészi magyar parazitája
}

András Kányádi

1 Né dans l'Antiquité, le type littéraire du parasite ne cesse de hanter le théâtre européen. Produit de la Renaissance, le théâtre hongrois valorise d'entrée de jeu cette figure. Dévorateur, provincial et sans scrupules : voilà les trois aspects fondamentaux du parasite qui domineront pour les siècles à venir les lettres magyares. Or le texte fondateur, qui remonte à une réécriture de Sophocle et réunit déjà ces trois traits, disparaît dans le tourbillon de l'Histoire pendant plus de trois cents ans. Quand il est enfin retrouvé au fond d'une bibliothèque allemande, le parasite a déjà fait son chemin comique sur les scènes européennes, et la réédition de la pièce exige des rajustements en conformité avec l'horizon d'attente d'un public habitué à ce personnage littéraire. Pourtant, il s'agit d'une figure complexe qu'on ne peut ramener à la seule fonction comique; dès lors, pour mieux comprendre son rôle, un examen plus approfondi s'avère nécessaire.

\section{La renaissance d'Électre}

Dans l'Europe centrale du $\mathrm{XVI}^{\mathrm{e}}$ siècle, l'union fort heureuse entre humanisme et protestantisme entraîne non seulement un intérêt pour l'érudition classique mais aussi l'essor de la création dans les langues vernaculaires. D'une part, le nombre des étudiants formés dans des centres prestigieux s'accroît; d'autre part, la réussite de la Réforme passe par les langues "vulgaires ». Le patrimoine antique se transmet, même si l'outil de la transmission est loin d'être parfait et, en quelques décennies, les ouvrages classiques traduits ou adaptés en allemand, tchèque, polonais, croate et hongrois se multiplient. Dans cette effervescence créatrice, un cas particulièrement 
fascinant est le remaniement de Sophocle par l'un des "pères" de la littérature hongroise, Péter Bornemisza.

3 Il faut dire tout de suite que depuis le début du $\mathrm{xvI}^{\mathrm{e}}$ siècle, les tragiques grecs deviennent largement accessibles grâce au latin : les manuscrits originaux sont assortis de commentaires latins ou bien traduits dans la langue d'Ovide. Si Érasme et Buchanan contribuent grandement à la notoriété d'Euripide, la renommée de Sophocle se propage aussi très vite: la première édition complète, parue à Venise, ouvre la voie à de nombreuses éditions commentées ${ }^{1}$. Mais il n'y a pas que le latin: traductions et adaptations dans différentes langues européennes voient désormais le jour. Les vedettes sophocléennes sont partout Ajax et Électre, mais les approches théâtrales diffèrent beaucoup d'un pays à l'autre.

4 Les uns essaient de rendre Sophocle d'une manière juxtalinéaire, tel Lazare de Baiff dans sa Tragédie de Sophocle intitulée Electra (de Baïf 1537). Le paratexte de l'ouvrage indique une traduction du grec,

en rythme françoyse, ligne pour ligne et vers pour vers, en faveur et commodité des amateurs de l'une et l'autre langue.

La visée moralisatrice de la préface de Baïf est on ne peut plus nette :

pour remonstrer aux roys et grans seigneurs l'incertitude et lubrique instabilité des choses temporelles : à fin qu'ils n'ayent confiance qu'en la seule vertu.

6 Les autres lettrés ne gardent en revanche que le canevas de Sophocle, tel l'Espagnol Fernan Pérez de Oliva, recteur de l'université de Salamanque. Sa pièce, écrite en 1528 et intitulée La venganza de Agamemnon (Oliva 1528), se fonde sur l'idée de la vengeance et Électre, assoiffée de sang, se montre prête à participer au massacre d'Égisthe. Il s'agit d'une version fortement christianisée de Sophocle, purgée des éléments mythologiques et arrosée de sang. Dans A vingança de Agamenom (Victoria 1536), le Portugais Anrrique Ayres Victoria prétend traduire du grec alors qu'il utilise surtout l'ouvrage de son contemporain castillan, dont il abrège légèrement le texte. Son mérite principal consiste à employer, contrairement à Pérez de Oliva qui préfère l'adaptation en prose, une forme portugaise très populaire à l'époque, le quintile heptasyllabique rimé. Quant à l'auteur italien Ludovico Martelli (1533), il prend encore plus de libertés en cultivant la contamination: sa Tullia (Martelli 1533) se présente comme un curieux amalgame entre Sophocle et Tite-Live, où la trame d'Électre se conjugue avec l'histoire du légendaire Tarquin le Superbe, dernier roi de Rome.

7 C'est dans ce contexte d'engouement pour la tragédie grecque que prend naissance la perle du drame magyar de la Renaissance, la Tragédie en langue hongroise d'après l'Électre de Sophocle ${ }^{2}$, appelée communément "Électre hongroise", de Péter Bornemisza. L'auteur, un prédicateur protestant, a une vie mouvementée : il perd ses parents au moment de la prise de Buda par les Turcs et grandit en Haute-Hongrie. Emprisonné à Kassa, il réussit à s'évader, fait des études à Padoue, Vienne et Wittenberg, et rentre au pays pour se mettre au service de la Réforme. Précepteur, imprimeur, prédicateur et auteur, il écrit les Postilles (Bornemisza 1573-1579), un volumineux recueil de sermons qui recèle aussi son sulfureux chef d'œuvre intitulé Les Tentations diaboliques (Bornemisza 1579). Son Électre est le fruit de son séjour viennois, où il suit les cours sur Sophocle du professeur helléniste Georg Tanner, qui était fortement influencé par la théorie du drame de Philipp Melanchthon ${ }^{3}$. C'est cette théorie que Bornemisza reprendra dans la postface érudite de l'édition viennoise de son ouvrage. 
Dans Cohortatio ad legendas tragoedias et commoedias 4 , Melanchthon (1546) développe l'idée que les auteurs grecs, en particulier Sophocle, illustrent le fonctionnement de la Providence: le péché des héros de la tragédie n'est que la violation de l'un des dix commandements, et cette infraction déchaîne la juste colère divine, suivie d'un terrible châtiment. Dans le cas de l'Électre sophocléenne, deux interdits sont enfreints, le cinquième et le sixième commandement, soit le meurtre et l'adultère ; la punition du couple royal devient ainsi inévitable et légitime. Dans sa préface à la réédition de la Chronique de Charion ${ }^{5}$ (Chronica 1533), Melanchthon explique que l'Histoire illustre les lois de Dieu ; il détaille amplement les leçons à tirer des histoires païennes par rapport aux deux commandements déjà cités: Hannibal et Brutus ont péri à cause d'un meurtre, David et les habitants de Troie ont péri, eux, à cause d'un adultère. Ce n'est donc pas par hasard si la postface de Bornemisza souligne que "quelle que soit l'époque, ce sont les mêmes châtiments qui attendent les assassins et les adultères " (p.592) et qu'il utilise une typographie spéciale pour faire ressortir les "règles" universelles :

les Erynnyes accompagnent toujours les péchés monstrueux ou

qui gladium acceperit, gladio peribit

9 figurent en capitales (p. 593). En tant que futur imprimeur, il va même rajouter à son livre deux illustrations : sur la première, on voit Caïn tuant Abel, la deuxième reproduit David apercevant Bethsabée.

Bornemisza affirme avoir utilisé le texte original de Sophocle, mais l'avoir modifié " pour embellir la pièce ». Il a aussi simplifié ou supprimé les personnages. Ainsi, le Chœur des vierges sophocléen se voit réduit à une seule femme, la vieille, du nom de Chorus. Le précepteur du drame grec a pour nom Maître et rappelle de près la figure d'un prédicateur. Pylade disparaît complètement, Égisthe, en revanche, se manifeste dès la première scène alors que chez Sophocle il ne surgissait qu'à la fin. Plusieurs scènes sophocléennes se trouvent écourtées ou renversées, les caractères du couple royal sont plus élaborés; le changement le plus curieux réside dans l'introduction d'une nouvelle figure, appelée Parasitus. Pour justifier sa présence dans la tragédie, plusieurs hypothèses peuvent être formulées. Contentons-nous ici d'en explorer cinq, en parcourant leur portée littéraire, morale, sociale, politique et rhétorique.

\section{Le flatteur térentien}

11 Parasitus semble être un personnage comique en raison de deux traits de caractère amplifiés: l'avidité et l'hypocrisie. Il apparaît en ouverture de la pièce, tenant compagnie à Égisthe qu'il ne cesse d'approuver, alors qu'en aparté il se moque de lui. Le mérite principal de son seigneur résiderait dans le gaspillage illégitime :

tel que je le vois, celui-là n'est pas Agamemnon, il sait user et abuser de la fortune

des autres (p. 548).

L'effet comique de ces paroles prend de l'importance dans le bref monologue du début, qui constitue une sorte de confession: Parasitus ne se soucie que de son bien-être personnel et cherche le moment propice pour soutirer à son maître « une chaîne en or d'une longueur de quatre aunes » (p. 550). Après tout, il se plaît à la cour parce qu'il y trouve son compte et que le roi usurpateur est, comme lui, hédoniste. Les deux scènes 
où il reparaît par la suite nous éclairent sur son côté zélateur. Au deuxième acte, sur l'ordre d'Égisthe, il fait seller les chevaux et rassembler la cour pour la chasse. Au cinquième acte, sur l'ordre d'Oreste, il accourt pour tuer son maître. Du reste, c'est dans cette scène finale que son hypocrisie atteint son comble: c'est une scène bouffonne, puisque le jeune vengeur l'apostrophe en l'appelant « son fidèle serviteur " et sollicite son aide. Parasitus se met en quatre pour plaire à son nouvel employeur : il lui fait des promesses :

Votre Altesse, je vais moi-même lui couper sa tête de traître, prodigue des conseils :

Ne le laisse pas caqueter, agis!

et insulte lui-même Égisthe :

Tais-toi, tu dois mourir ! (p. 588).

Bornemisza a lu les auteurs de la comédie attique, en particulier Térence, puisqu'il parle dans ses Postilles de la vantardise exprimée par « les trasos et les parasites et tous ces fanfarons » (p. 888). Cela renvoie directement aux héros de l'Eunuque, Thraso, le soldat fanfaron et Gnatho, son parasite. Dans Électre, l'auteur recycle admirablement ces deux figures térentiennes: si Égisthe et Parasitus sont inséparables, c'est pour marquer la lâcheté des imposteurs. Qu'on songe, à nouveau, à la définition de Melanchthon formulée dans sa préface à Térence :

tragoedia habent laeta principia et tristes exitus, comoedia autem tristia principia et laetos exitus (Melanchthon 1546).

Il s'agirait alors d'une astuce dramaturgique. Mais une autre source moins théorique semble aussi avoir influencé la conception du personnage : la comédie de Hans Sachs, intitulée Persones ${ }^{6}$ (Sachs 1558). La confidente royale s'y appelle Parasita, elle a pour tâche de séduire Aristote, ce qui nécessite beaucoup d'éloquence. Dans la Tragédie de Clytemnestre $^{7}$ (Sachs 1554) de ce prolifique maître nurembergeois, on tombe sur un couple de trésoriers ${ }^{8}$ - Dion et Cleon - qui, commentateurs inlassables des agissements de leurs maîtres, sont récompensés par la reine avec « ein marck goltds ». Serait-ce le motif de l'or ou celui de la profession qui les apparente au héros hongrois ? Quoi qu'il en soit, Parasitus fait sans cesse l'inventaire des choses, comme un véritable trésorier : " douze rois », « soixante porte-drapeaux », " quatre aunes de chaîne en or ». Fruit de la technique dramatique classique ou de l'intertextualité contemporaine, Parasitus porte indiscutablement le sceau de la littérarité.

\section{Le courtisan profiteur}

15 Avec Parasitus, Bornemisza campe un type social répandu de son époque, celui du courtisan profiteur. Grâce à ce visage, son héros rejoint la grande famille shakespearienne qui va de l'inoffensif Rosencrantz au dangereux Oswald. Chez l'auteur hongrois, la tragédie grecque est transposée dans l'ambiance d'une cité de la Renaissance. Il y a l'urbanisme caractéristique de l'époque : Égisthe se réjouit de ses «forteresses solides » et de ses "villes au trésor ». On retrouve la pratique de la chasse - Parasitus donne ses instructions au "grand veneur" - mais aussi celle du divertissement musical à la cour des seigneurs, avec l'inventaire de rigueur des instruments de l'époque: tambours, luths, violons, trompettes, flûtes à bec. Les vêtements (draps, velours dorés) dialoguent avec le mobilier (buffets remplis 
d'argenterie), mais aussi avec les pratiques sexuelles: Électre se plaint d'être traitée " comme une bâtarde ", Clytemnestre critique les maigres performances au lit de feu son mari. On n'occulte donc rien, si ce n'est pour comploter.

Dans cette ambiance d'allégresse générale, le seigneur de Mycènes mène une vie somptueuse et le courtisan partage volontiers ce faste. Cependant, si Égisthe se place sous le signe de la réjouissance, Parasitus, plus circonspect, guette la conjoncture favorable :

dans sa joie, il n'y a rien que je ne puisse lui demander, je vais m'affairer autour de lui (p. 550).

C'est en bon connaisseur de la nature fugace de la générosité qu'il fait venir les musiciens. Et c'est toujours avec le même instinct d'orientation qu'il change de camp au bon moment, se ralliant à Oreste et retournant les armes contre son maître. Conscient de l'attente envers sa prise de position, il n'hésite pas à accuser Égisthe de «trahison " et se montre impitoyable. Au personnage comique se superpose donc ici le personnage social qui agit sous la contrainte de la hiérarchie, au nom du pouvoir « légitime ». Par son geste " dévoué », Parasitus consolide, voire regagne, sa place dans le nouvel ordre qui est en train de se construire.

Perfide mais réaliste, flatteur mais lucide : il faut aussi préciser que le mode de vie du courtisan demande beaucoup de souplesse car l'époque est en pleine ébullition. Dans la Hongrie du seizième siècle, de grands bouleversements s'opèrent sur le plan historique : à la suite de la bataille de Mohács et de la prise de Buda ${ }^{9}$, le royaume est divisé en trois parties - le Sud et le Centre sont occupés par les Ottomans, le Nord et le Nord-Ouest deviennent la possession des Habsbourg et le Nord-Est avec la Transylvanie ont un roi hongrois ${ }^{10}$. Dans ce royaume affaibli, des seigneurs parfois très influents font la loi, et le courtisan, pour survivre, doit s'adapter aux caprices des puissants. Par temps de guerre, la dépendance économique s'avère cruciale : Parasitus s'efforce, en fin de compte, de capitaliser ce que son maître prodigue jette par la fenêtre. C'est un collectionneur qui sait compter et qui sait aussi tuer pour avoir la vie sauve.

\section{Le justicier patriotique}

La pièce de Bornemisza comporte une longue postface en latin où l'auteur explique pourquoi il a choisi l'Électre de Sophocle ${ }^{11}$. D'après lui, cette tragédie soulève une question politique majeure : faut-il tuer le tyran

quand la patrie souffre dans la plus atroce servitude (p. 597)

ou laisser le temps adoucir les maux ? Cette question est évoquée dans le dialogue entre Électre et Chrysothémis, la première incarnant la révolte ouverte, la seconde la passivité tactique. Nous avons déjà esquissé la division de la Hongrie à cette époque. L'auteur, qui a souffert à la suite de l'occupation ottomane de Buda et a été emprisonné par les Habsbourg, a choisi sans doute ce sujet héroïque pour indiquer le chemin des armes: Mycènes gémit sous le joug d'Égisthe, tout comme la Hongrie sous l'ennemi, et la libération s'avère salutaire. Dans cette optique, la figure ambiguë et souple de Parasitus serait inspirée par un personnage historique, connu pour sa versatilité politique mais aussi pour ses prouesses militaires.

21 Menyhért Balassa ${ }^{12}$, un puissant hobereau de la Haute-Hongrie, changeait de camp comme de chemise. Tantôt au service du roi hongrois, tantôt fidèle à la maison des 
Habsbourg, il cumulait ses possessions et ses titres au gré du plus offrant. En 1558, l'année de naissance de l'œuvre de Bornemisza, il a joué un rôle important dans le démantèlement d'une conspiration en Transylvanie, ce qui lui a valu le titre de gouverneur de cette région. Bornemisza connaissait bien sa famille, il allait même devenir le précepteur de son neveu ${ }^{13}$, et peut-être voyait-il dans ces revirements l'excuse d'un camouflage intentionnel car, en son for intérieur, Balassa s'opposait à Ferdinand et était de religion protestante. Vu sous cet angle, les répliques de Parasitus concernant le rétablissement de la justice seraient fondées : il s'agirait alors d'un allié d'Oreste qui tient en haute estime le roi Agamemnon et qui songe depuis toujours à le venger. Le moment venu, il jette son masque et sa brutalité n'est que l'expression d'une haine de longue date à l'égard de l'imposteur. Cela expliquerait aussi le choix de l'auteur d'avoir renoncé à la figure de Pylade dont la présence chez Sophocle, bien que muette, servait à rendre la vengeance solennelle et héroïque.

La postface de Bornemisza tranche avec la pièce : c'est une dissertation savante, peutêtre un travail de mémoire à l'université de Vienne, mené sous la direction de Georg Tanner. La question politique que l'étudiant hongrois qualifie de si «centrale » chez Sophocle ne l'est pas autant dans son adaptation. Mais le contexte d'instabilité dans laquelle la tyrannie d'Égisthe avait plongé sa cité émerge maintes fois au cours de l'histoire universelle. Les Mouches (1943) de Sartre thématise la France sous l'Occupation, Le Deuil sied à Électre de O'Neill ${ }^{14}$ (1931) se déroule dans une Amérique affaiblie par la Guerre de Sécession. Le chaos qui règne dans la Hongrie du seizième siècle n'est-il pas un sujet qui, à lui seul, soulève des interrogations politiques? Dans cette optique, Parasitus devient un patriote engagé pour la libération de son pays, dont la stratégie principale est la dissimulation.

\section{Le catholique dépravé}

Bornemisza est, avant tout, un prédicateur de la Réforme. Sa culture humaniste ne l'empêche pas de prôner la justice divine et la traduction ne cache pas sa véritable intention : réécrire Sophocle, le christianiser - la pièce est destinée «à améliorer les mœurs chrétiennes». Ce but est poursuivi avec rigueur tout au long du texte. L'exemplification dans le prologue est nette :

Dieu n'oublie pas le péché et, plus il tarde à intervenir, plus son châtiment sera terrible, sans égard à la personne (p. 544).

Le Maître, créé à partir du précepteur sophocléen, ressemble à un prédicateur qui attire sans arrêt l'attention sur l'ampleur du châtiment divin. Oreste lui-même s'adresse à Dieu (et pas à Apollon) sous forme d'un genre religieux spécifique, la lamentation (Pirnát 1969). La moralité de la pièce, énoncée par le même Maître, ne laisse aucun doute :

Qu'il vive sa vie de manière pieuse et chaste s'il veut éviter le sort de ses ancêtres (p. 589).

Le « il » signifie Oreste, mais en fait vise tous les êtres humains. La postface disserte longuement sur le crime de l'adultère, la violation du cinquième commandement, en invoquant aussi bien les lettres de Saint Paul que l'éloge de la pudeur du poète païen Bacchylide. Et derrière chaque adultère, l'auteur croit découvrir l'activité du diable qui, dans un enchaînement inexorable, pousse l'humanité au meurtre. 
26 Au-delà de la visée moralisatrice quelque peu martelée, on peut se demander pourquoi Parasitus, qui méprise son seigneur, s'enflamme à l'idée du festin. S'il fait venir les musiciens, ce n'est pas seulement dans l'espoir d'obtenir la grosse chaîne en or, mais aussi parce qu'il aime se divertir. De toute évidence il adore, lui aussi, le luxe banni sous le règne parcimonieux d'Agamemnon dont il ne regrette pas la fin. En revanche, Électre, fille de son père, déteste les banquets ; le Maître les voit aussi d'un très mauvais œil et Oreste suit son précepteur qui prône la tempérance. S'opposent ainsi deux camps avec des habitus différents : d'un côté, l'exubérance catholique ${ }^{15}$, féodale, qui vit ses derniers jours de splendeur; de l'autre côté, le puritanisme protestant, capitaliste et austère, qui va bientôt s'emparer du pouvoir.

Un sous-texte biblique semble aussi hanter la pièce de Bornemisza : le topos du festin de Balthazar, décrit dans le livre de Daniel, cher aux auteurs protestants ${ }^{16}$. Rappelons-en quelques motifs : le roi veut récompenser Daniel, son serviteur, avec une chaîne en or ; le banquet d'allégresse ; l'énumération des instruments de musique ; les prémonitions sur la chute du royaume; la mort de Balthazar en clôture. On retrouve ces motifs chez l'auteur hongrois, la plupart d'entre eux étant liés directement à la figure de Parasitus. Il n'est pas alors interdit de penser que l'auteur avait l'intention d'écrire un travestissement du prophète de l'Ancien Testament, et ce pour mieux fustiger un milieu hongrois dépravé, désagrégé et catholique, en lui opposant le pasteur authentique, la figure du Maître. Il y a peut-être une invitation à la conversion au luthérianisme, bien que discrète, puisqu'à l'heure de l'écriture de la pièce, Bornemisza se trouve à Vienne, au milieu d'une majorité catholique. Mais quelle que soit la confession des héros, le désastre du pays est perçu comme un châtiment divin.

\section{Le prophète déchaîné}

Si l'on examine le discours de Parasitus, une particularité rhétorique ne peut nous échapper : ses répliques sont autant de prémonitions métaphoriques. D'entrée de jeu, il s'exclame :

Qu'on festoie tant qu'on puisse festoyer ! (p. 547).

29 À première vue, il s'agit d'une flagornerie vis-à-vis du mode de vie débridé mené par Égisthe, mais à la lumière de l'épigraphe du livre, ce passage revêt un tout autre sens. L'épigraphe en question est tirée de l'Évangile selon Luc:

Malheur à vous qui êtes repus maintenant, car vous aurez faim! Malheur à vous, qui riez maintenant, car vous serez dans le deuil et dans les larmes ! ${ }^{17}$.

Ainsi, Parasitus lancerait au tyran un avertissement voilé.

Dans un deuxième temps, il ordonne au grand veneur de sonner le cor pour que les chiens se rassemblent (p. 558).

$31 \mathrm{Du}$ coup, cette phrase se lit comme un jugement de valeur, désignant métaphoriquement la canaille de la cour qui ne vit que pour se divertir. Une métaphore qui n'annonce rien de bon. En rupture avec son entourage, Parasitus sera, dans la scène finale, l'instrument de la punition divine. Prêt à tuer Égisthe, il le menace :

Je lui avais dit qu'il regretterait son royaume,

32 le raille :

Tu n'es plus en mesure de donner des ordres,

incite le vengeur à accomplir son acte : 
Il cherche des échappatoires, ne l'écoute pas, annonce l'inévitable :

Tu dois mourir de sa main et tire les conclusions par l'entremise d'un proverbe qui utilise la métaphore canine :

Celui qui a agi en chien aura une récompense de chien (p. 588).

Son dernier geste indique le passage à l'acte : il saisit l'usurpateur par les cheveux et invite Oreste à lui «trancher la gorge ». Parasitus démontre une violence verbale digne des imprécations prophétiques et finit par aller même au-delà, en succombant à la tentation de l'agression physique.

Dans la postface, Bornemisza s'excusait de ne pas avoir développé une analyse aussi fine de la pièce que Tanner, son professeur, qui avait d'abord ajusté l'Électre sophocléenne aux enseignements moraux et politiques, pour en faire ensuite une démonstration de l'elocutio antique. Quant à lui, il n'a cherché, dit-il, qu'à

rendre l'intention du poète de la manière la plus simple et la plus claire (p. 598).

Cette excuse fait partie de l'ethos de l'orateur dans le but de gagner son audience par la modestie de son entreprise. Le véritable enjeu de cette adaptation est culturel :

En suivant l'exemple des nations plus cultivées, il nous faut cultiver et enrichir

chaque jour, et de mieux en mieux, la langue hongroise ${ }^{18}$.

C'est pourquoi les personnages parlent la langue du peuple, avec des adages et des mots parfois crus, mais d'une façon authentique et imagée; ils ouvrent la voie à l'épanouissement rhétorique de la langue magyare que les auteurs catholiques du baroque vont bientôt perfectionner.

\section{La postérité de Parasitus}

La vie posthume de cette pièce est passionnante car, malgré quelques mentions sporadiques chez des gens de lettres des XVIII et ${ }^{\mathrm{e}}$ xIX ${ }^{\mathrm{e}}$ siècles, le texte n'est découvert qu'en 1923, à la Bibliothèque de Gotha en Allemagne ${ }^{19}$. La critique hongroise se montre aussitôt dithyrambique, et un grand auteur de l'entre-deux-guerres, Zsigmond Móricz, en fait une adaptation (!), jouée avec succès au théâtre. Móricz, à qui on doit aussi le nouveau titre «Électre hongroise $»^{20}$, opère quelques changements dans la dramaturgie, en truffant la pièce de didascalies et de plusieurs rajouts archaïsants ; il modifie aussi le personnage de Parasitus. Chez lui, celui-ci devient «Pique-assiette » (tányérnyaló, littéralement Lèche-assiette), et son rôle de confident du couple seigneurial, en particulier de la reine, s'accroît. Si une partie de ses répliques dérive du texte original hongrois, il récite aussi les paroles de l'adaptateur, surtout dans ses dialogues avec la reine, absents chez Bornemisza. Contrairement à Parasitus, ce n'est pas Pique-assiette qui participe à l'exécution de l'usurpateur, mais Pylade. Móricz, tout en réhabilitant le personnage sophocléen, le transforme en une sorte de Fortinbras : Égisthe poignarde Oreste dans le dos, et Pylade, après s'être vengé, se fait couronner. Malgré l'enthousiasme de Móricz à l'égard du texte de son prédécesseur, qu'il qualifie de

premier livre du nouveau testament de la littérature hongroise (Móricz 1930), 
41

La contamination et l'adaptation, techniques de réécriture répandues à la Renaissance, arrivent aussi dans la Hongrie du $\mathrm{xvI}^{\mathrm{e}}$ siècle. Culture humaniste et religion protestante y font bon ménage. Si Péter Bornemisza se propose de traduire l'Électre de Sophocle, c'est dans un but de christianisation et d'enrichissement de la langue hongroise. Sa grande trouvaille réside dans la figure du parasite qui, malgré ses apparitions sporadiques, révolutionne la pièce par le poids qu'il prend au fil de l'intrigue. D'héritage térentien, il représente le courtisan profiteur de son époque mais rappelle autant une figure historique connue que le type du catholique luxurieux. Outil de la vengeance divine, Parasitus s'apparente également aux prophètes de l'Ancien Testament, quoique de manière caricaturale. Plus ténébreux que comique, il finit par dévorer son seigneur. Il est indubitablement notre contemporain.

\section{BIBLIOGRAPHIE}

BAïF Lazare de, 1537, Tragédie de Sophocles intitulée Electra, contenant la vengence de l'inhumaine et très piteuse mort d'Agamemnon roy de Mycène la grant faicte par sa femme Clytemnestra et son adultère Egistus, Paris.

BORNEMISZA Péter, 1558, Tragoedia magyar nyelven az Sophocles Electrájából, Vienne : Hoffhalter.

BORzSÁK István, 1960, „Az antikvitás 16. századi képe”, Bornemisza-tanulmányok, Budapest :

Akadémiai.

CARION Johannes, 1533, Chronica durch M. Johan. Carion vleissig zusamen gezogen, meniglich nützlicvh zu lesen, Wittemberg.

HUBAY Miklós, 1983, A dráma sorsa, Budapest : Szépirodalmi.

MARTELLI Ludovico, 1533, Tullia, Florence.

MELANCHTHON, 1546, Cohortatio ad legendas tragoedias et commoedias, in Joachim CAMERARIUS, P.

Terentii comoediae sex, Leipzig.

MÓRICZ Zsigmond, 1931, Magyar Elektra.

MÓRICZ Zsigmond, 1930 „Bornemisza Péter Electrája”, Nyugat, nº 24, p. 809-822.

NEMESKÜRTY István, 1959, Bornemisza Péter az ember és az író, Budapest : Akadémiai.

oliva Fernan Pérez de, 1528, La venganza de Agamemnon. Tragedia cuyo argumento es de Sofocles, poeta griego, Burgos.

Études finno-ougriennes, 46 | 2014 
PIRNÁT Antal, 1969, „A magyar reneszánsz dráma poétikája”, Irodalomtörténeti közlemények, nº 5, Budapest.

SACHS Hans, 1554, Tragedia mit 14 personen. Die mördisch königin Clitimestra, und hat 5 actus, Nuremberg.

SACHS Hans, 1558, Persones, die königin, reit den philosophum Aristotelem, Nuremberg.

SZIGETI József, 1967, „A Balassi comoedia és szerzője”, Irodalomtörténeti füzetek, n 54, Budapest :

Akadémiai.Victoria Anrrique Ayres, 1536, A vingança de Agamenom, Porto.

\section{NOTES}

1. Sophoclis tragoedia septem cum commentariis, Venise, 1502 ; Sophoclis tragoedia septem cum interpretationibus vetustis et valde utilibus, Florence, 1522; Sophoclis tragoedia septem cum commentariis interpretationum argumenti Thebaidos fabularum Sophoclis, Haguenau, 1534; Interpretatio tragoaediarum Sophoclis ad utilitatem iuventutis quae studiosa est Graecae linguae, Francfort, 1549.

2. Bornemisza Péter, Tragoedia magyar nyelven az Sophocles Electrájából, Hoffhalter, Vienne, 1558. La pièce a été écrite à l'occasion du couronnement de Ferdinand de Habsbourg, le 8 juin 1558. Pour les citations, nous utilisons le volume Heltai Gáspár és Bornemisza Péter művei, Budapest: Szépirodalmi 1980, p. 541-599.

3. Sur le séjour viennois de Bornemisza cf. István Nemeskürty 1959. Sur l'influence de Tanner, cf. Antal Pirnát 1969 et István Borzsák 1960. Tanner, qui a fini ses études à Wittenberg en 1546, a certainement assisté à la célèbre conférence que Melanchthon a faite sur l'Électre de Sophocle à l'université de Wittenberg, en 1545.

4. C'est la préface de Melanchthon à l'édition de l'œuvre de Térence par Joachim Camerarius.

5. Cette chronique, très populaire à l'époque, a eu de nombreuses rééditions. La préface de Melanchthon apparaît dans l'édition de 1558.

6. C'est István Borzsák qui attire l'attention sur ce personnage dans ses études sur Bornemisza (1960).

7. Cf. l'édition d'Adelbert von Keller (éd.), Hans Sachs, Tübingen, Laupp, 1874, vol. 9, p. 317-341. p. 4, note 12. Chez Sachs, c'est Érigone, la fille d'Égisthe qui cherche à se venger, mais le meurtre d'Oreste ayant été jugé légitime, elle se pend. Influencé par Boccace, Sachs écrira un autre texte sur le sujet, dans lequel Clytemnestre sera définie comme « adultère criminelle » (Sachs 1558).

8. Sachs utilise le mot « kemmerlig », ce qui signifie trésorier (lat. camerarius).

9. En 1526, l'armée ottomane écrase les Hongrois à la bataille de Mohács. Le roi hongrois Louis II y trouve la mort et le pays se désagrège, même si la capitale, Buda, ne sera prise qu'en 1541.

10. Jusqu'en 1571, Jean Sigismond est le roi reconnu par une partie de la noblesse hongroise. Après sa mort, la Transylvanie devient principauté, soutenue par le sultan, et Ferdinand reste le roi du Royaume de Hongrie.

11. La postface s'intitule «Salutem benigno lectori » et explique les méthodes utilisées par Melanchthon et par Tanner dans l'interprétation de l'Électre de Sophocle. 
12. Menyhárt Balassa (1511 ?-1568, Vienne). Cette théorie du modèle historique apparaît dans l'étude de József Szigeti, J.Sz., „A Balassi comoedia és szerzője”, Irodalomtörténeti füzetek, no. 54, Budapest: Akadémiai, 1967.

13. Ce neveu, Bálint Balassi, allait devenir le plus grand poète de la Renaissance hongroise.

14. Eugene O'Neill, Mourning becomes Electra (1931).

15. Idée lancée par Miklós Hubay 1983, „Magyar Elektra” (1949).

16. Cf. Daniel, $5: 1-30$.

17. Cf. Luc $6: 25$.

18. Cet exemple de l'édification de la langue sera repris par le Dalmatien Dominko Zlatarić, dans la préface qu'il fait à sa traduction croate, Elektra, Venise, 1598.

19. Le 12 juillet 1923, le directeur de la Bibliothèque de Gotha, M. Krüger, écrit une lettre à l'Académie des Sciences Hongroises dans laquelle il annonce qu'au cours de l'inventaire, les bibliothécaires ont trouvé un texte hongrois. La pièce de Bornemisza a paru en fac-similé le 17 novembre 1923, à Budapest. Le seul exemplaire connu du texte original continue d'enrichir la bibliothèque allemande.

20. Móricz Zsigmond, 1931. Le texte original de Bornemisza ne sera joué qu'en 1946.

\section{RÉSUMÉS}

La redécouverte humaniste des œuvres classiques de l'Antiquité a une incidence considérable sur les littératures nationales à l'époque de la Renaissance. Aussi l'Électre sophocléenne connaît-elle de nombreuses traductions et adaptations. Parmi ces dernières, on trouve la pièce de Péter Bornemisza, le premier drame important en langue hongroise, qui reflète aussi bien la théorie dramatique protestante de Melanchthon que l'influence des comédies de Térence. Notre étude tente d'analyser les différents aspects de la meilleure trouvaille de l'auteur de l'Électre hongroise, la figure de Parasitus. Courtisan profiteur ou prophète déchaîné, la complexité du parasite magyar soulève des interrogations littéraires, morales, sociales, politiques et rhétoriques.

The rediscovery of the ancient European literary legacy plays a prominent role in the Renaissance of national language literatures. Sophocles' Electra undergoes several translations and adaptations, among them that of Péter Bornemisza, the author of the first important Hungarian language drama, which combines Melanchton's protestant spirit with the comedies of Terence. Our paper attempts to analyse the main literary, moral, social, political and rhetorical aspects of Parasitus, the most original character in the 'Hungarian Electra'.

A humanisták által újrafelfedezett ókori klasszikusok művei jelentős szerepet játszanak a nemzeti nyelveken íródó reneszánsz irodalmakban. Szophoklész Elektrájának számtalan fordítása és átdolgozása keletkezik, köztük Bornemisza Péteré, aki Melanchton protestáns szellemben fogant drámaértelmezését Terentius vígjátékaival ötvözve alkotja meg az első jelentős magyar nyelvű drámát. Tanulmányunk a „Magyar Elektra” legkiválóbb szerzői leleményét, a Parasitus-figura irodalmi, erkölcsfilozófiai, társadalmi, politikai és retorikai vonatkozásait vizsgálja. 


\section{INDEX}

disciplines allemand, castillan, croate, français, grec, hongrois, latin, polonais, portugais, tchèque

Mots-clés : adaptations, humanisme, théâtre, protestantisme, traductions

Keywords : Adaptations, humanism, theatre, protestantism, translations, literature, Castillan, Croatian, Czech, French, German, Greek, Hungarian, Latin, Polish, sixteenth century, Twentieth century, Hungarians, Turks, Portuguese, Buda, Germany, Gotha, Kassa, Mohács, Padua,

Salamanca, Transylvanie, Venice, Vienna, Wittenberg, Antiquity

nomsmotscles Hongrois, Ottomans

Index géographique : Allemagne, Buda, Gotha, Kassa, Mohács, Padoue, Salamanque, Transylvanie, Venise, Vienne, Wittenberg

Thèmes : littérature

Index chronologique : antiquité, XVIe siècle, XXe siècle 